the guinea pig, although feet, claws, and vagina are pigmented in some individuals. (b) The process of infection occurs mainly in the basal layers of the superficial (as opposed to follicular) epidermis, so that the hairs borne by artificially blackened white skin tend to remain white, even after manipulative treatment (such as grafting itself) which causes the hairs to be thrown off and replaced by new. We believe that this is because the primordia of new hairs are usually formed from the bases or shafts of the old follicles, to which the infective agent does not gain access (possibly because the rate of upward flow or migration of follicle epithelium is greater than the rate of spread of infection downwards). But black hairs do sometimes form in artificially blackened white epithelium; as often, it may be, as the primordia of new hairs are budded off from the blackened superficial epithelium rather than from the epithelium of effete follicles. (c) Contrary to the belief of Fessler ${ }^{6}$, skin grafts, competently transplanted, invariably grow hairs. (d) The rate of blackening of a white-in-black graft seems to vary directly with the intrinsic (that is, 'graft-specific') rate of cell division in the epidermal graft. It is particularly high in sole-of-foot and claw-forming epithelia, and white grafts of such epithelia blacken in a matter of weeks. The rate of centripetal blackening of a white dorsal skin graft in a black skin area is, however, anomalously slow : we do not yet know why. (e) The pigmented character of artificially blackened epithelia of all types is a property of all the cells of their basal layers (cf. Fig. B). Whatever may be the role of melanophores in amphibian skin grafts, we have no evidence that they play any primary part in the phenomena we have described.

Summary. Black guinea pig epidermis has the power to 'infect' neighbouring white epidermis, and so to blacken it. This is shown not to be due to a cellular invasion and a replacement of white cells by black. It is due to an agent which, having entered white cells, brings about a permanent heritable change that causes them and their descendants to remain thereafter black. The infective agent may be a self-reproducing body housed normally in the cytoplasm ; or, less probably, it may be a morphogenetic hormone that merely initiates the formation of such self-reproducing bodies in the white cells which it infects. The experiments described have a direct bearing on the problems of somatic cellular differentiation and heredity. ${ }^{2}$ Carnot, P., and Deflandre, C., C.R. Soc. Biol. Paris, 48, 178, 430
(1896).

"Loeb, L., Arch. EntwMech. Org., 6, 1 (1897).

"Loeb, L., "The Biological Basis of Individuality", pp. 14, 141 (Springfeld, 1945). Kelley, R. W., and Loeb, L., Anat. Rec., 74, 487 (1939)

'The view adopted by Saxton, J. A., Schmeckebier, M. M., and Kelley, R. W., Biol. Bull., '71, 453 (1936); Fessler, A., Brit. J. Dermatol., 53,' 201 (1941); but rejected by Lewin, M. L., and Peck, S. M., J. Invest. Dermatol., 4, 483 (1941).

- Our operation was based on the description of claw anatomy by le Gros Clark, W. E., Proc. Zool. Soc., 1, 1 (1936).

- Fessler, A., Brit. J. Dermatol., 53, 201 (1941).

'Cf. Claude, A., Science, 97, 451 (1943).

- Lindegren, C. C., Ann. Mo. Bot. Gard., 32, 107 (1945); Proc. Nat. Acad. Sci., 32, 68 (1946).

- Spiegelman, S., Ann. Mo. Bot. Gard., 32, 139 (1945).

${ }^{10}$ Darlington, C. D., Nature, 154, 164 (1944).

${ }^{11}$ Haddow, A. M., Nature, 154, 194 (1944).

"Sonneborn, T. M., Proc. Nat. Acad. Sci., 29, 329, 338 (1943).

${ }^{12}$ Medawar, P. B., Brit. Med. Bull., 3, 70 (1945).

"Cf. Needham, J., "Biochemistry and Morphogenesis", 267 (Cambridge, 1942),

"Sale, L., Arch. EntwMech. Org., 37, 248 (1913). Seelig, M. G., Arch.

${ }^{16}$ Medawar, P. B., J. Anat., Lond., '78, 176 (1944); 79, 157 (1945).

\section{FUNDAMENTAL PROBLEMS IN MODERN PHYSICS}

$T$ HE Physical Society intends to hold a series of ordinary meetings at places outside London, and the first of these took place in the Physics Department of the University of Birmingham on December 20. This meeting, which took the form of a one-day conference under the general title "Fundamental Problems in Modern Physics", was attended by about two hundred fellows of the Society and visitors from universities, government establishments and industrial firms. The morning session was devoted to three papers; in the first, Prof. R. E. Peierls, of the University of Birmingham, discussed some of the outstanding difficulties of fundamental theoretical physies, and indicated the type of experimental evidence which would be most valuable in resolving them. He was followed by Mr. J. S. Gooden, also of the University of Birmingham, who read a paper on the design of a proton synchrotron, with special reference to the factors influencing the amplitude of phase oscillations of the particles and consequently affecting the intensity of the emergent beam. The third paper, by Mr. D. W. Fry, of the Telecommunications Research Establishment, dealt with some practical aspects of synchrotron design, in the light of experience gained with the three electron synchrotrons already under construction in Great Britain. A paper by Prof. J. Sayers, of the University of Birmingham, on recombination phenomena in highly ionized gases, opened the afternoon session; Prof. Sayers was unfortunately not able to be present at the meeting, but his paper was read by Prof. P. B. Moon. The conference closed with a paper by Dr. F. Hoyle, of Cambridge, on the synthesis of heavy elements from hydrogen in stars, and their distribution throughout space.

Prof. Peierls suggested three main lines of experimental investigation from which information useful to the theoretical physicist may be forthcoming. In the first of these, the energies of the nuclear particles involved are not excessive, and the greatest need is for improvement of measuring apparatus and for a systematic collection of results. In the case of $\beta$-disintegration, for example, it is now known that some of the apparent discrepancies between experiment and the original Fermi theory were due to experimental difficulties which have since been overcome; but there still remains some freedom of choice in formulating the theory, which can be narrowed down only by detailed knowledge of quantities such as the spin and symmetry of the initial and final nucleus and the distribution in energy of the emitted particles.

With regard to the short-range forces between particles in complex nuclei, Prof. Peierls pointed out that although Bohr's 'liquid drop' model represents the qualitative properties of such nuclei reasonably well, the assumptions made are independent of the nature of the forces involved, and hence the deductions drawn from them can give no information on this point.

Even if the laws of force were known, it would be impossible to calculate exactly the values of the energy-levels of complex nuclei, on account of the large number of particles involved. We must therefore discuss these nuclei in a statistical way, and so it becomes important to have a large amount of experimental data systematically collected. The 
importance of each individual item might seem small, but that of the whole would be considerable if an effort were made to obtain as much information as possible about each energy-level, and great care were taken to proceed systematically in those cases where it is necessary to obtain information from transitions between two levels.

The difficulties with which the theoretical physicist has to contend in describing nuclear processes while still ignorant of the law of force between the particles are augmented by doubts as to the limits of applicability of the fundamental mechanical laws. For example, an unstable condition is predicted on extrapolating to short distances the law expressing the non-central force between the components of the deuteron. Experimental data on neutron-proton collisions, when the bombarding particle has a de Broglie wave-length of the same order of magnitude as the distance at which the simple law breaks down, would therefore be of value in suggesting suitable modifications for use at these distances. Particles with kinetic energy in the region of $12 \mathrm{MeV}$., or greater if possible, would be required, and accurate data from the scattering experiments are essential. Turning to experiments in which very high energies are required, Prof. Peierls pointed out that existing theory breaks down completely when applied to particles the distances of approach of which are of the order of $10^{-13} \mathrm{~cm}$., and to states existing for the order of $10^{-23}$ sec. To provide a signpost for theory in this region, the study of collisions between elementary particles at energies of the order of $100 \mathrm{MeV}$. is necessary. Another possible outcome of experiments on particles with energies in the $100 \mathrm{MeV}$. region is the production of mesons in controllable experimental conditions, whereupon it might be possible to investigate their electric charge, to ascertain whether they have a range of masses, and to obtain further data on their spin, in circumstances more amenable to interpretation than those existing at present, when the only source of mesons is cosmic radiation.

In the two papers which followed, Mr. Gooden and Mr. Fry took up Prof. Peierls' challenge to practical physics by showing how high-energy particles might be obtained. Referring to the proton synchrotron under construction at the University of Birmingham, Mr. Gooden showed that in the case where the particle velocity, and consequently the frequency of the accelerating radio-frequency field, increases with increasing particle energy, some particles will possess a phase oscillation the amplitude of which is to a first approximation constant, and might, under certain conditions, steadily increase. If the amplitude is allowed to increase, these particles enter the region of unstable phase, and are lost from the circulating beam. Particles can, however, be accelerated provided that a careful control is exercised in the choice of those physical variables which affect the amplitude of the oscillation. Mr. Gooden showed that there are no less than eight significant forces affecting this oscillation, four of which cannot be varied. Three of these are associated with the radial displacement of a particle from the central, or stable, orbit, since such displacements determine the energy gain per revolution required to maintain the particle in its instantaneous orbit, the amount of magnetic flux enclosed by the orbit, and the response of the particle to changes in the frequency of the accelerating electric field. The fourth invariable force arises from the fact that, as the total kinetic energy of the particle increases, the radial displacement resulting from the given change in the energy-gain per revolution becomes smaller. The combined effect of these four forces is to produce a very small damping of the phase oscillation in the case of acceleration of protons to energies of about $1,000 \mathrm{MeV}$. The four variable forces were then discussed; these were shown to depend on the rate of change of the frequency and amplitude of the accelerating field, the form of time variation adopted for the magnetic field, and the shape of the faces of the accelerating chamber. Finally, Mr. Gooden considered the factors affecting the number of radio-frequency cycles, and the time interval per cycle, during which particles could be accepted for acceleration.

Mr. Fry restricted his attention to electron synchrotrons, and therefore discussed only those types of machine in which the initial acceleration (to velocities of about $0.98 c$ ) is obtained by betatron action, the radio-frequency accelerating pulse being phased to start just as the betatron core begins to become saturated. When the particle velocity is nearly equal to that of light, the orbital frequency will remain constant if the radius of the orbit is kept constant by suitable increase of the magnetic field with time; the frequency of the alternating voltage between the accelerating electrodes can then be constant during each pulse. After mentioning the essential components of a synchrotron system and the relative times at which the voltages must be applied to the injection gun, accelerating electrodes and ejection coils, Mr. Fry went on to describe the magnet systems used in the $30 \mathrm{MeV}$. machine designed by the Telecommunication Research Establishment and the $300 \mathrm{MeV}$. synchrotron which is being made by Metropolitan-Vickers with help from the Establishment for the University of Glasgow. To reduce the magnet weight, it is convenient to use a symmetrical iron circuit of high-quality laminations in which a high flux density can be achieved. Considerations of the maximum energy which has to be stored in the field show that it is desirable to keep the volume of the annulus in which the particles circulate as small as possible; this, in turn, necessitates very careful design and construction to prevent particles from being lost on account of irregularities or out-ofphase fields produced in air gaps by leakage from the magnet stampings. In the case of the $300 \mathrm{MeV}$. machine, the stored energy is supplied by a condenser bank; but the high cost of this equipment makes it necessary to consider alternative sources, such as a motor generator or a resonant magnet circuit working on a reduced duty cycle, to supply higher energy synchrotrons. Mr. Fry concluded his paper with a discussion of the type of resonator which it is proposed to use in the radio-frequency system, in order to minimize eddy current field, the technical problems associated with making the vacuum envelope for the $300 \mathrm{MeV}$. machine, and the arrangements for obtaining a high injection voltage (about $100 \mathrm{kV}$.) prior to betatron acceleration.

Prof. Sayers's paper began with a summary of the measurements by Kenty on the decay of ionization in argon after the sudden interruption of a lowvoltage arc. Later measurements by Mohler in cæsium and mercury vapour were similar to Kenty's, in that mechanical commutators were used to interrupt the arc and apply voltages after various intervals to Langmuir probes, by which the electron densities were measured. Sayers used a cathode-ray oscilloscope to measure the current of a Langmuir probe, main- 
tained at an adjustable potential, as a function of time after the interruption of a low-voltage are in argon. From a series of such measurements the timevariation of the electron (or ion) concentration and the electron temperature could be determined at various places in the tube. The loss of particles by diffusion to the walls was illustrated by graphs showing the concentration as a function of position within the tube at different times; at first the concentration was practically uniform over the greater part of the tube, but this region of uniformity became more and more closely confined around the axis of the tube as time went on. Within this region, loss by diffusion may be neglected, so that the value of $\alpha$, the coefficient of recombination, may be deduced from the equation $\frac{d N}{d t}=-\alpha N^{2}$, where $N$ is the number per eubic centimetre of ions of either sign. Sayers found that $\alpha$ varies with electron temperature, but is independent of the pressure over the range $0 \cdot 1-1 \cdot 0 \mathrm{~mm}$., as would be expected if the recombination is a two-body process. At an electron temperature of $1,000^{\circ} \mathrm{K}$., $\alpha$ for argon is about $6 \cdot 6 \times 10^{-10} \mathrm{~cm}^{3} \mathrm{sec}^{-1}$.

In comparing these experiments with theory, Prof. Sayers pointed out that the probability of recombination to the ground state of the neutral atom is likely to be only a small fraction of the total recombination probability and, since the excited states will be roughly hydrogen-like for all atoms, it is not unreasonable to compare calculated values of $\alpha$ for oxygen with the experimental values obtained for argon, mercury and cæsium. The variation of $\alpha$ with electron temperature is as predicted by theory, but the absolute magnitude of $\alpha$ is about five hundred times larger than theory predicts. Observations on recombination in the $F$ region of the ionosphere agree with the laboratory experiments, but considerations of the balance of electron population in interstellar space agree with the theoretical value. In contrast to these unresolved discrepancies concerning the cap. ture of electrons into the whole series of states of the atom, measurements by optical methods of the radiative capture of electrons into individual atomic states seem to agree well with theory.

The discussion which followed centred largely around the question of whether, in the laboratory experiments, it is proper to consider the electrons as being in temperature equilibrium at each instant during the few milliseconds occupied by the decay of the ionization.

In the last paper of the afternoon session, Dr. Hoyle discussed on $\Theta$ of the most fascinating problems of astrophysics-the process of synthesizing heavy elements from hydrogen and helium, and obtaining the observed distribution of these elements throughout space. There are two main problems to be considered : first, it is necessary to establish the conditions in which this synthesis can take place, and secondly to determine where, in the universe, these conditions are actually to be encountered. Having considered the very great difficulties involved in any theory which envisages conditions other than those of thermal equilibrium between the different elements, Dr. Hoyle proceeded to discuss the results obtained by applying statistical mechanics to the evolution of the heavy elements. He pointed out that from this point of view nuclear reactions can be treated by applying the well-known properties of statistical assemblies of dissociating gases, together with an equation determining the neutron-to-proton ratio as a function of density. This latter equation shows that both the concentration of free neutrons and the ratio of neutrons to protons increase with increasing density, and that to obtain a proportion of heavy elements in accordance with observation, densities of the order of $10^{7} \mathrm{gm}$. per $\mathrm{cm}^{3}$ are required. It can be shown also that to establish equilibrium between the various elements in a time sensibly less than the age of the universe, the region considered must have a temperature of the order of $10^{\circ}$ degrees. The central temperatures of normal stars are too low by a factor of about 100, but in the case of those which have collapsed to a small fraction of their initial radius owing to the exhaustion of their hydrogen supply, temperatures of the required magnitude may be encountered. At some stage during the collapse, depending on the angular momentum, which differs widely from one star to another, the rotating spheroid becomes unstable, and material is thrown off into space from its equator. Now the nuclear reactions which would have to take place in order to reestablish equilibrium at the lower temperatures and densities require the emission of energy, so cooling is rapidly accelerated. Dr. Hoyle suggested that a small fraction of the heavy elements formed in a collapsed star remain 'frozen'. Thus, since the amount of material thrown off may amount to 99 per cent of the total mass of the star, such elements may be present both in the material thrown off and in the residual core. Dr. Hoyle connected the super-nova catastrophe with physical conditions inferred from the hypothesis that the Crab nebula represents the remains of a past super-nova.

Among difficulties raised in the course of the discussion was that of accounting for the presence of heavy elements in all known stars, which contain also hydrogen and helium.

\section{THE GIACOBINID METEOR SHOWER, 1946}

GEVERAL papers describing British observations of the Giacobinid meteor shower, 1946, were presented at a meeting of the Royal Astronomical Society on December 13, 1946. Special interest was attached to this subject because of the incidence of a shower of such exceptional intensity at a time when the considerable advances made by radar techniques were able to make an important contribution to the observations. Such methods not only enabled estimates of hourly rates to be made without interference due to poor visibility, but also demonstrated how information on certain meteor characteristics can be provided more conveniently and more precisely than is possible by visual meteor watches.

An introductory paper on the visual watch was given by J. P. M. Prentice, director of the Meteor Section of the British Astronomical Association. Although observations were considerably hindered by bad weather, it was possible to obtain a complete curve showing the variation in hourly rate by com. bining the results of several observers. The shower was of approximately six hours duration, rising steeply to a sharp maximum around $0345 \mathrm{hr}$., followed by a steep decline. The variation in rates was so rapid that the mean hourly rates, determined by averaging over short periods, are considerably affected by the length of period chosen. The visual observa. tions were averaged over 7-8 minutes, and the maximum hourly rate corrected to a radiant in the 